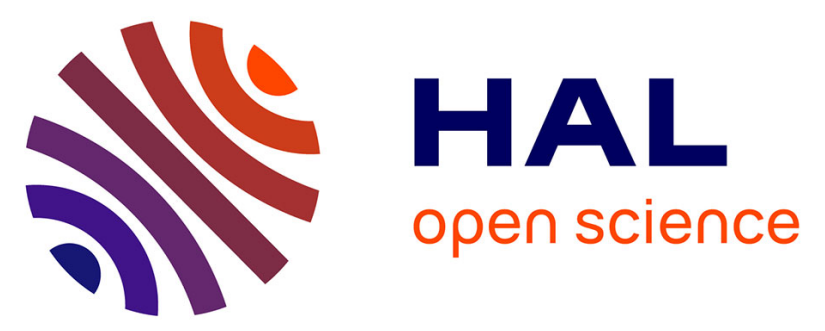

\title{
Dynamic equilibria and magnetohydrodynamic instabilities in toroidal plasmas with non-uniform transport coefficients
}

\author{
Shimpei Futatani, Jorge Morales, Wouter J.T. Bos
}

\section{To cite this version:}

Shimpei Futatani, Jorge Morales, Wouter J.T. Bos. Dynamic equilibria and magnetohydrodynamic instabilities in toroidal plasmas with non-uniform transport coefficients. Physics of Plasmas, 2015, 22, pp.052503. 10.1063/1.4919960 . hal-01298324

\section{HAL Id: hal-01298324 \\ https://hal.science/hal-01298324}

Submitted on 8 Apr 2016

HAL is a multi-disciplinary open access archive for the deposit and dissemination of scientific research documents, whether they are published or not. The documents may come from teaching and research institutions in France or abroad, or from public or private research centers.
L'archive ouverte pluridisciplinaire HAL, est destinée au dépôt et à la diffusion de documents scientifiques de niveau recherche, publiés ou non, émanant des établissements d'enseignement et de recherche français ou étrangers, des laboratoires publics ou privés. 


\title{
Dynamic equilibria and magnetohydrodynamic instabilities in toroidal plasmas with non-uniform transport coefficients
}

\author{
Shimpei Futatani, ${ }^{1}$ Jorge A. Morales, ${ }^{2}$ and Wouter J. T. Bos ${ }^{1}$ \\ ${ }^{1}$ LMFA-CNRS UMR 5509, Ecole Centrale de Lyon, Université de Lyon, 69134 Ecully, France \\ ${ }^{2}$ CEA Cadarache, St. Paul Lez Durance, France
}

(Received 13 February 2015; accepted 27 April 2015; published online 21 May 2015)

\begin{abstract}
It can be shown that in the presence of a toroidal magnetic field induced by poloidal coils, combined with the electromagnetic field induced by a central solenoid, no static equilibrium is possible within the MHD description, as soon as non-zero resistivity is assumed. The resulting dynamic equilibrium was previously discussed for the case of spatially homogeneous resisitivity. In the present work, it is shown how a spatial inhomogeneity of the viscosity and resisitivity coefficients influences this equilibrium. Parameters in both the stable, tokamak-like regime and unstable, reversed field pinch-like regime are considered. It is shown that, whereas the magnitudes of the velocity and magnetic field fluctuations are strongly modified by the spatial variation of the transport coefficients, the qualitative flow behaviour remains largely unaffected. (C) 2015 AIP Publishing LLC.

[http://dx.doi.org/10.1063/1.4919960]
\end{abstract}

\section{INTRODUCTION}

Plasmas confined by toroidal magnetic and electric fields are generally considered the best candidates to achieve sustainable nuclear fusion. Tokamaks and Reversed Field Pinches (RFPs) are two of such configurations which are currently investigated intensively. In the ideal case, the plasmas in such reactors would remain quietly confined within the magnetic field in order to allow their core to reach the temperature needed for thermonuclear fusion. Unfortunately such a quiet confined state is, in general, not observed, and both turbulent small scale motion and collective bulk motion lead to a complicated dynamics which is not easily modeled. The coarsest description of such plasmas taking into account a number of key features of realistic plasmas is the magnetohydrodynamic (MHD) description. MHD does not take into account effects induced by the local violation of charge-neutrality in the plasma, a source of small-scale turbulent fluctuations, in particular, at the edge, but it does take into account the global dynamics of the bulk of the plasma, including kink and tearing instabilities. Understanding the detailed MHD behaviour of a toroidal plasma in a toroidal magnetic field is therefore a prerequisite for a successful development of more complex plasma descriptions. However, even on the level of MHD, the current understanding of toroidal plasmas is incomplete. In the past, an important number of numerical studies of the RFP configuration have considered the simplified case of a cylindrical geometry. For instance, the influence of the Hartmann number (which we will define below) on the onset of instabilities in the RFP was considered in Refs. 1-3, the effect of compressibility in Refs. 4 and 5. And, even though the curvature of the magnetic field induces effects absent in cylindrical geometry, ${ }^{6}$ recent studies in cylindrical geometry show some agreement with experiments. ${ }^{7,8}$

To study tokamaks, MHD has also been widely applied, see, for instance, Refs. 9-11. In the older literature, it has been common use to start a tokamak description considering a force-free static equilibrium. Such an equilibrium only exists if the pressure forces are balanced by the Lorentzforces that originate from the imposed magnetic and electric fields. In straight cylinder configurations, such force-free states can be easily defined, considering, for instance, z-pinch and $\theta$-pinch devices. ${ }^{12}$ Even though such geometries can be subject to MHD instabilities, a force-free state can be defined. This changes in toroidal geometry.

In the simplest case in which the toroidal electric field is generated by a central solenoid, without external current drive, and where the toroidal magnetic field is induced by the poloidally orientated coils, ignoring ripples and other details, the imposed electro-magnetic fields have a simple form. It was shown in previous studies ${ }^{13-16}$ that for this case, assuming uniform electric resistivity, such an equilibrium is not possible. These studies showed, following an increasing level of complexity, that the velocity can never be zero if the current density is linked to the electric field by Ohm's law. The present work builds upon the results of these studies, increasing by one step the complexity, considering spatially non-uniform electric resistivity and viscosity profiles. Indeed, in practice, strong pressure, density, and temperature gradients will influence the local values of the viscosity and resistivity in the plasma. The present approach takes this into account in the coarsest way, by defining profiles as a function of the minor radius. In particular, we will show how different types of dynamic equilibrium and MHD instabilities appear as a function of the value and the spatial distribution of the transport coefficients.

In Sec. II, we will outline the MHD description which we consider, and we will explain how we choose the externally imposed electromagnetic fields. In Sec. III, we present the results of simulations which show how the electric resistivity profile influences the dynamics when compared to a uniform resistivity in both RFP and tokamak regime. Conclusions are given in Sec. IV. 


\section{MHD EQUATIONS, MAGNETIC GEOMETRY, AND NUMERICAL METHOD}

\section{A. Equations}

In the magnetohydrodynamic approximation, plasma is modeled as a charge-neutral electro-magnetic conducting fluid. In the incompressible description, the dynamics of the velocity $\boldsymbol{u}(\boldsymbol{x}, t)$ and $\boldsymbol{B}(\boldsymbol{x}, t)$ are given, in Alfvénic units, by

$$
\begin{gathered}
\frac{D \boldsymbol{u}}{D t}=-\nabla p+\nabla \cdot \boldsymbol{\sigma}+\boldsymbol{j} \times \boldsymbol{B} \\
\frac{\partial \boldsymbol{B}}{\partial t}=-\nabla \times \boldsymbol{E}, \\
\boldsymbol{E}=\eta \boldsymbol{j}-\boldsymbol{u} \times \boldsymbol{B}, \\
\nabla \cdot \boldsymbol{u}=0, \\
\nabla \cdot \boldsymbol{B}=0,
\end{gathered}
$$

with $D / D_{t}$ denoting the material derivative, the current density $\boldsymbol{j}=\nabla \times \boldsymbol{B}$, the pressure $p$, the electric field $\boldsymbol{E}$, and the resistivity $\eta$. All the quantities in these equations are made dimensionless using the Alfven speed $C_{A}=B_{0} / \sqrt{\rho \mu_{0}}$ as a reference velocity, with $\rho$ the density and $\mu_{0}$ the magnetic permeability constant. The stress tensor $\sigma_{i j}$ is given by

$$
\sigma_{i j}=\nu\left(\frac{\partial u_{i}}{\partial x_{j}}+\frac{\partial u_{j}}{\partial x_{i}}\right)
$$

with $\nu$ the kinematic viscosity. In the case of a spatially uniform viscosity and density and resistivity, Eqs. (1) and (2) simplify to

$$
\begin{gathered}
\frac{D \boldsymbol{u}}{D t}=-\nabla p+\nu \Delta \boldsymbol{u}+\boldsymbol{j} \times \boldsymbol{B}, \\
\frac{D \boldsymbol{B}}{D t}=\boldsymbol{B} \cdot \nabla \boldsymbol{u}+\eta \Delta \boldsymbol{B} .
\end{gathered}
$$

The study of the difference in dynamics induced by the simplification leading from (1) and (2) to (7) and (8) is the main subject of the present investigation. Therefore, the following two cases will be considered:

(I) Uniform magnetic resistivity $\eta_{0}$ and viscosity $\nu_{0}$;

(II) Space dependent magnetic resistivity $\eta(r)$ and viscosity $\nu(r)$.

These two cases will be indicated by the roman capitals $I$ and $I I$ in the following.

\section{B. Magnetic topology}

In our study, the plasma is confined in a toroidal domain with a circular poloidal cross-section. We assume the toroidal magnetic field to be induced by poloidal coils, ignoring the ripples induced by the nonhomogeneous distribution of the latter. This leads to a toroidal magnetic field proportional to

$$
B_{\text {tor }} \sim \frac{1}{R}
$$

The toroidal electric field is deduced from the assumed presence of a central solenoid aligned with the main axis of the torus, through which the magnetic flux is a linearly increasing function of time, so that the electric field is also inversely proportional to the major radius

$$
E_{\text {tor }} \sim \frac{1}{R}
$$

In the absence of velocity, the profile of the current density can be easily determined through Ohm's law (3)

$$
\boldsymbol{j}=\boldsymbol{E} / \eta
$$

If the magnetic resistivity is uniform, the toroidal current induced by the solenoid will then also satisfy (10). In the case of a non-uniform resistivity, the current profile will be different. The viscosity and resistivity profiles we consider in the present work mimic the profiles induced by the strong drop of temperature near the edge of experimental fusion reactors and are given by

$$
\eta(r)=\eta_{0} f(r) \quad \nu(r)=\nu_{0} f(r),
$$

with

$$
f(r)=\left(1+\left(\frac{r}{a}\right)^{2 \Lambda}\right)^{1+1 / \Lambda}
$$

with $\Lambda=4{ }^{1}$ This resistivity profile, combined with Eq. (11), yields the current density profile shown in Fig. 1(a). To compare the two cases, we have fixed the total amount of the imposed toroidal current to be equal for both cases, i.e.,

$$
I_{0}=\int j_{\eta(r)} d S=\int j_{\eta_{0}} d S
$$

where the integration is performed over a poloidal cross-section.

The combination of the toroidal magnetic field with the toroidal current, together with its induced poloidal magnetic field, leads to a Lorentz force $\boldsymbol{j} \times \boldsymbol{B}$ which is not curl-free. It can therefore not be balanced by the pressure gradient (since its curl is zero) and necessarily velocity fields emerge. ${ }^{13}$ These velocity fields can be of steady nature, in which case one could call them dynamic equilibria. ${ }^{14-16}$ In certain cases, MHD instabilities can lead to a chaotic, or even turbulent state, and the parameters which delimit the thresholds to this state were previously shown to be the Hartmann number and the pinch-ratio. ${ }^{1-3}$ The Hartmann number represents the ratio of electromagnetic forces to viscous forces, and is defined as

$$
H a=\frac{B_{0} L}{\sqrt{\nu \eta}},
$$

where $L$ is a normalization lengthscale and $B_{0}$ a reference value of the magnetic field. It is clear that in the case of nonuniform transport coefficients, $\mathrm{Ha}$ is inhomogeneous as well. Its profile as a function of the major radius is shown in Figure 1(b). In this work, the Prandtl number

$$
\operatorname{Pr}=\frac{\nu}{\eta},
$$

is set to unity everywhere. This implies that the resistive and viscous Lundquist-numbers, $S, M$, respectively, 


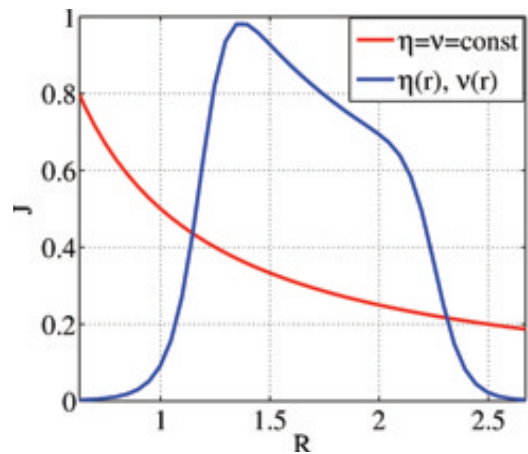

$$
S=\frac{B_{0} L}{\eta}, \quad M=\frac{B_{0} L}{\nu},
$$

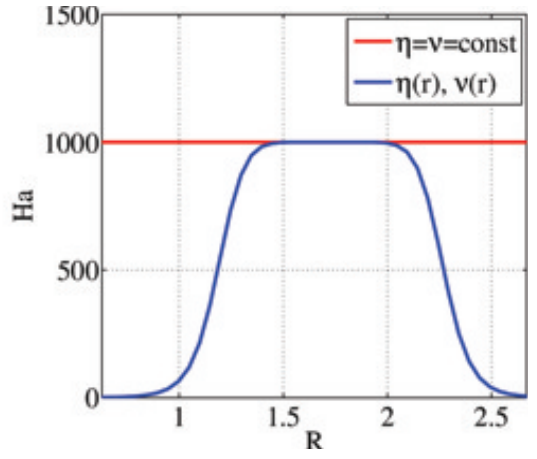

$$
\Theta=\frac{\overline{B_{p o l}(a)}}{\left\langle B_{t o r}\right\rangle},
$$

with $a$ the minor radius, the bar indicating an average over the surface with $r=a$, and the brackets indicating a volume average. MHD instabilities generally appear when both the pinch-ratio and the Hartmann number are large enough. Judging from Figure 1(b), for case II, MHD instabilities are thus expected to be more easily observed in the core than in the edge of the plasma. In tokamak geometries, the toroidal field is generally stronger than the poloidal field, and the pinch ratio is consequently small. In this regime, where
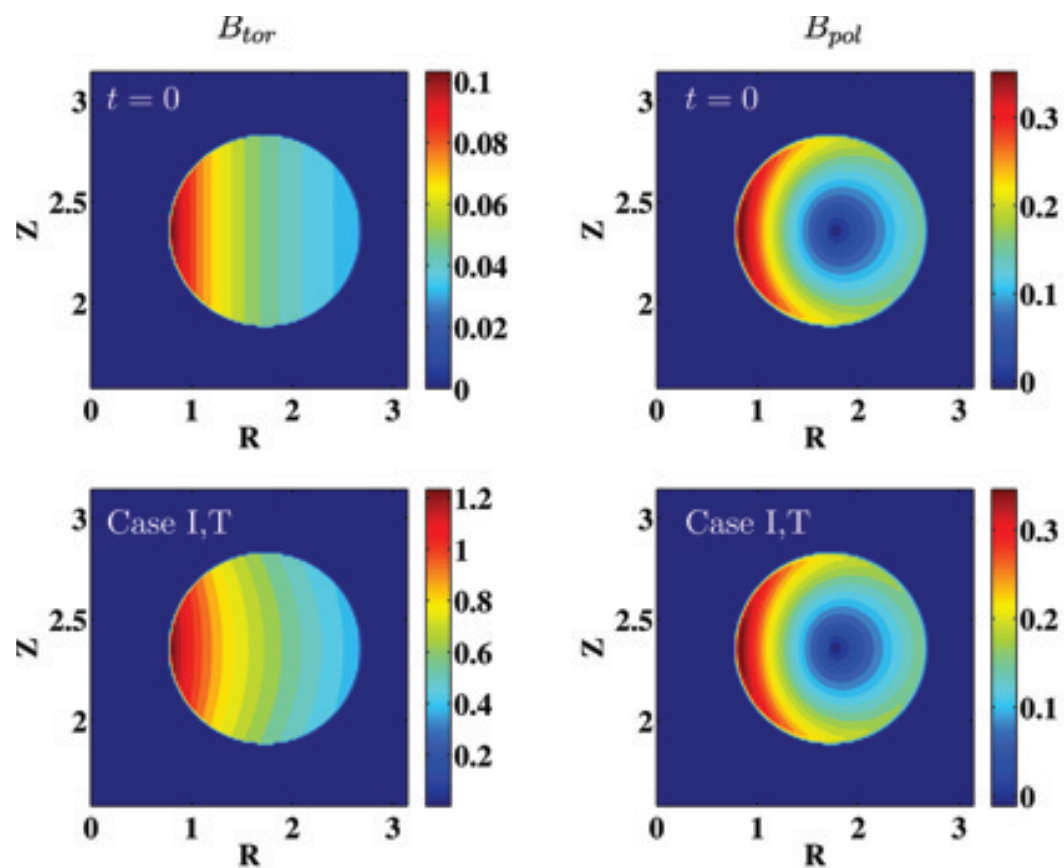

FIG. 2. Poloidal cross section showing isovalues of the magnetic fields for $M$ $=2000$ and uniform transport coefficients. On the top row, we show the externally imposed magnetic fields, generated by the combined effects of the toroidal magnetic and electric fields. On the center and bottom rows, we show the steady-state profiles for the tokamak and RFP regimes.
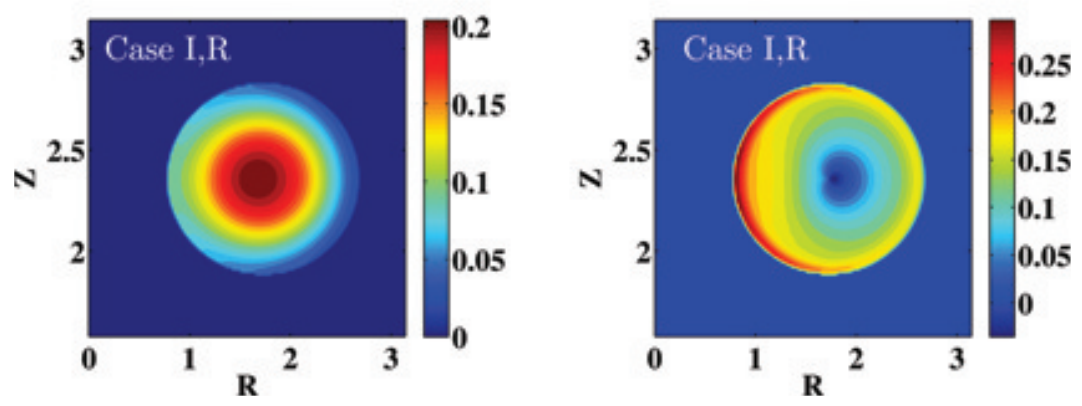

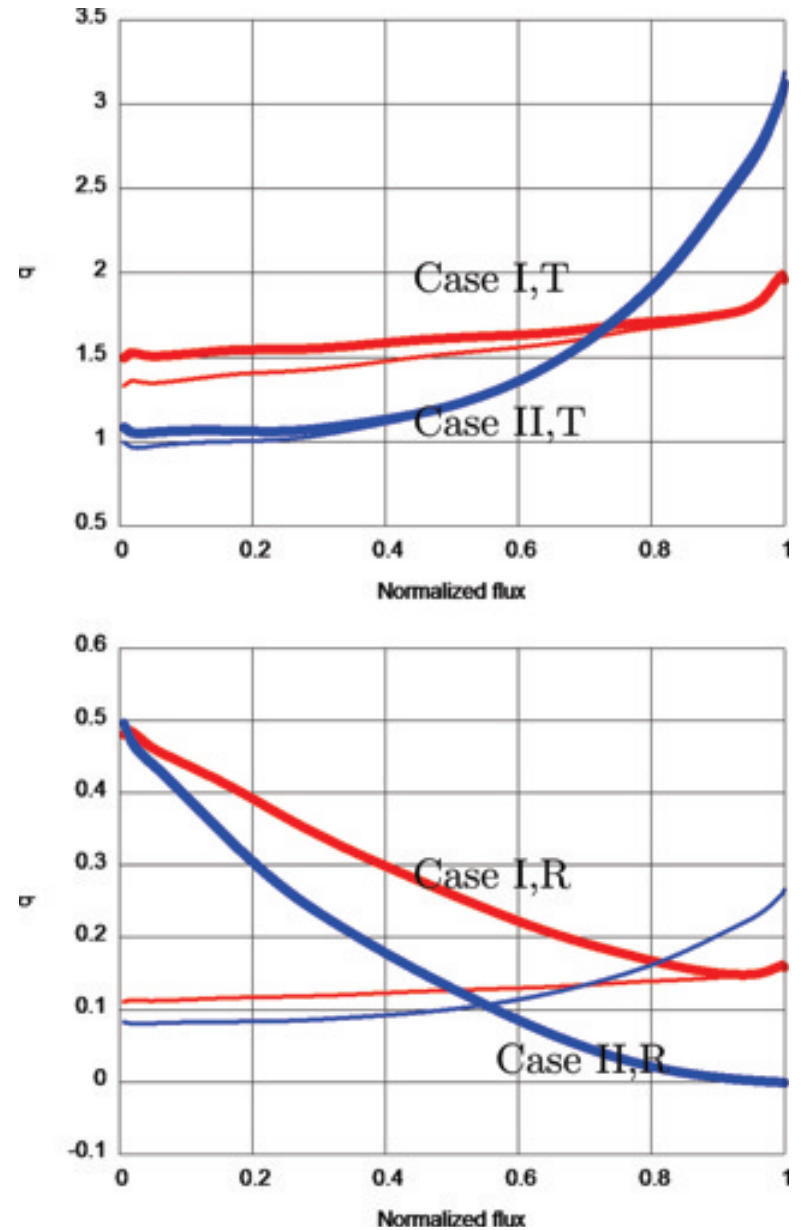

FIG. 3. Profiles of safety factor $q$ versus the flux-function for the different cases. Thin lines show the initial q-profile while thick lines indicate the final state. Red lines correspond to case I, blue lines to case II.

MHD instabilities are avoided, one usually uses the safety factor to characterize the magnetic field geometry. This parameter, roughly related to the inverse of the pinch ratio, is defined by

$$
q=\frac{1}{2 \pi} \oint \frac{1}{R} \frac{B_{t o r}}{B_{p o l}} d l
$$

where the contour is taken in the poloidal plane. This contour can be defined as a circle at fixed minor radius, but instead of the minor radius, the safety factor is generally plotted as a function of the flux function, since the magnetic surfaces are not perfectly axisymmetric around the toroidal axis. The normalized flux, which sets the flux function to unity at the separatrix (the last closed flux surface of the plasma) is defined as

$$
\psi_{\text {norm }}=\frac{\psi-\psi_{0}}{\psi_{\text {sep }}-\psi_{0}}
$$

where $\psi_{0}$ is the minimum magnetic flux at the core plasma and $\psi_{\text {sep }}$ is the magnetic flux at the separatrix. In our case, we choose the separatrix to be just inside the boundary of the plasma domain.
In all our simulations, the toroidal geometry has a minor radius $a=0.3 \pi$ and a major radius $R_{0}=0.55 \pi$; the aspect ratio is $R_{0} / a \sim 1.83$. The geometry is the same as in Ref. 6 , and details on the generation of the magnetic field can be found in Ref. 17.

\section{Numerical method and boundary conditions}

The simulations have been performed with a pseudospectral MHD solver which is based on the volume penalization method. ${ }^{18}$ In hydrodynamics, immersed boundary techniques, including penalization approaches, are nowadays commonly employed to solve boundary or initial-boundary value problems in complex geometries. Recently, these methods have been extended to treat magnetohydrodynamic flows. ${ }^{19}$ Those methods consist in embedding the original, possibly complex spatial domain inside a larger domain having a simpler geometry, for example, a Cartesian geometry, while keeping the boundary conditions approximately enforced-thanks to the new terms that are added to the equations. In this work, the immersed boundary technique is applied to produce the toroidal geometry. The size of the periodic box where the calculations are performed is $(2 \pi \times 2 \pi \times \pi)$. The boundaries are enforced by adding a term of the form of a Darcy-drag, $-(\chi / \epsilon)\left(\boldsymbol{u}-\boldsymbol{u}_{0}\right)$ to the right hand side of the Navier-Stokes equations (1). Here, $\chi$ is a mask function which is unity in the boundaries and zero in the plasma. The vector $\boldsymbol{u}_{0}$ is the velocity that is imposed in the boundaries, and $\epsilon$ is a permeability parameter which is taken small, in the present case $\epsilon=5 \times 10^{-4}$. The wall is thereby modeled by a porous medium where the permeability is taken small enough to neglect the velocities in the walls. The mathematical convergence of the method was studied in Ref. 18. For the magnetic field, we impose the magnetic field at the wall in a similar fashion, ${ }^{17,19}$ by adding a term $-(\chi / \epsilon)\left(\boldsymbol{B}-\boldsymbol{B}_{0}\right)$ to the RHS of the induction equation (2). The boundary conditions for the velocity are no-slip at the walls, corresponding to $\boldsymbol{u}_{0}=0$. For the magnetic field, we use the same value of $\epsilon$ to impose Dirichlet boundary conditions at the wall. These conditions for the magnetic field are the following: We set the wall-normal component of the magnetic field zero at the wall. This implies that there is no magnetic flux through the wall. The poloidal component of the magnetic field at the wall is determined such that it corresponds to the magnetic field induced by the imposed toroidal current. The third component of the magnetic field, in the toroidal direction, is left free. This degree of freedom is important in the present case since the value of the toroidal magnetic field can change drastically in the RFP regime. The initial conditions are zero for the velocity field and the perturbations of the magnetic field. From those quiescent initial conditions, a non-trivial velocity field is generated, interacting with the magnetic field, leading in most simulations to a dynamic steady state. In the RFP limit, this state can change into a multi-mode or turbulent state as will be observed. The resolution for all calculations is $256^{3}$ grid points.

\section{SIMULATION RESULTS}

The full characterization of toroidally confined magnetofluids necessarily involves the exploration of an enormous 

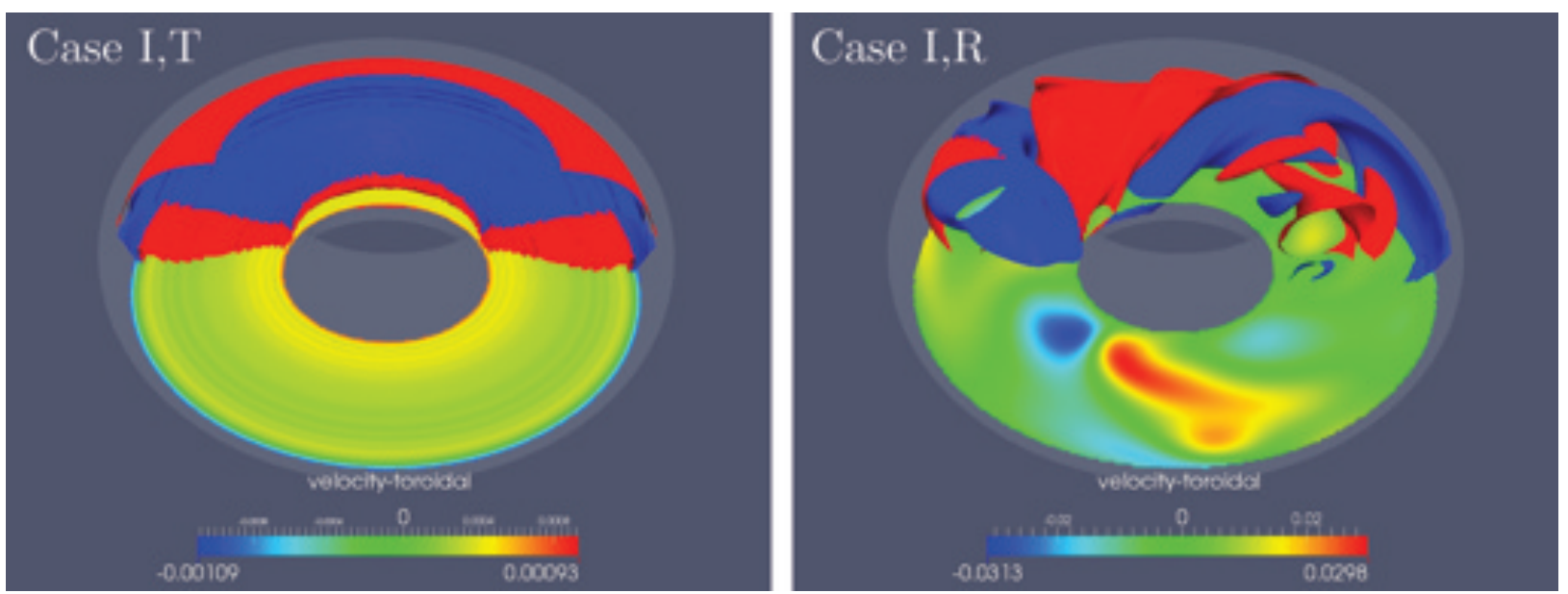

FIG. 4. Isosurfaces for a value of $20 \%$ of the maximum toroidal velocity (red contours show positive velocity and blue contours negative) at steady state for Lundquist number $(M=2000)$. Also, a cross-section at a fixed value of the vertical coordinate is shown, quantifying the toroidal velocity in the plane.

parameter space. Any investigation aiming at the understanding of the influence of the variation of a certain parameter on the dynamics should carefully choose how the remaining parameters are fixed. For practical reasons, in particular, due to the computational cost of the simulations carried out, the Lundquist number is limited to a few thousand. Even though this value is low compared to realistic values in existing machines, the results might be fairly relevant if one considers that the effective transport coefficients are in reality greatly influenced by micro-turbulence, thereby reducing the effective Lundquist number by orders of magnitude. The calculations are performed at Lundquist numbers ranging from $10^{2}$ up to $M=3000$, and most detailed results will be presented for $M=2000$. For this last value of $M$, we investigated values for the toroidal magnetic field, $B_{\text {tor }}=1.2,0.6$, $0.4,0.3,0.1,0.05$, while keeping the toroidal current, and thereby the poloidal magnetic field, constant. At high enough pinch ratios, which corresponds in our case to low enough toroidal magnetic field strength (more precisely for $B_{\text {tor }}<0.1$ for $M=2000$ ), a threshold is crossed towards an unstable region of parameter space. The MHD (kink) instability

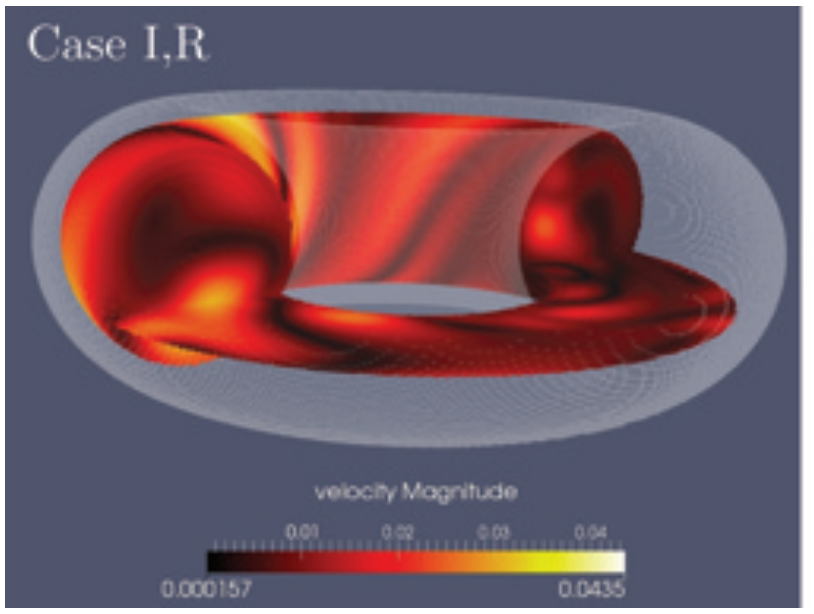

triggers a velocity field which significantly modifies the magnetic field. This latter dynamics is typical of RFP devices. For the low values of the pinch ratio, the velocity field is not zero, but dynamic, non-chaotic equilibria are observed. In the following, we will call the chaotic state the RFP regime and the non-chaotic dynamic equilibria the tokamak regime. All results are presented during the statistically stationary state of the system.

In the presentation of the results, we will indicate by Case $I$ the simulations where we assumed space-uniform constant magnetic-resistivity $\eta_{0}$ and kinematic viscosity $\nu_{0}$. The other, Case II, corresponds to the plasma where we have taken into account the non-uniform values of $\nu(r)$ and $\eta(r)$. For both cases, the highest pinch-ratio case, where $B_{\text {tor }}$ $=0.05$ (case I), $B_{\text {tor }}=0.1$ (case II), will be called the Rregime (for RFP) and the opposite, $B_{\text {tor }}=0.6$ (case I), $B_{\text {tor }}=$ 1.2 (case II) the T-regime (for tokamak). Let us stress that this nomenclature is chosen with respect to the presence, or absence, of MHD instabilities, without any claim that the dynamics are closely reproducing the dynamics in actual machines.

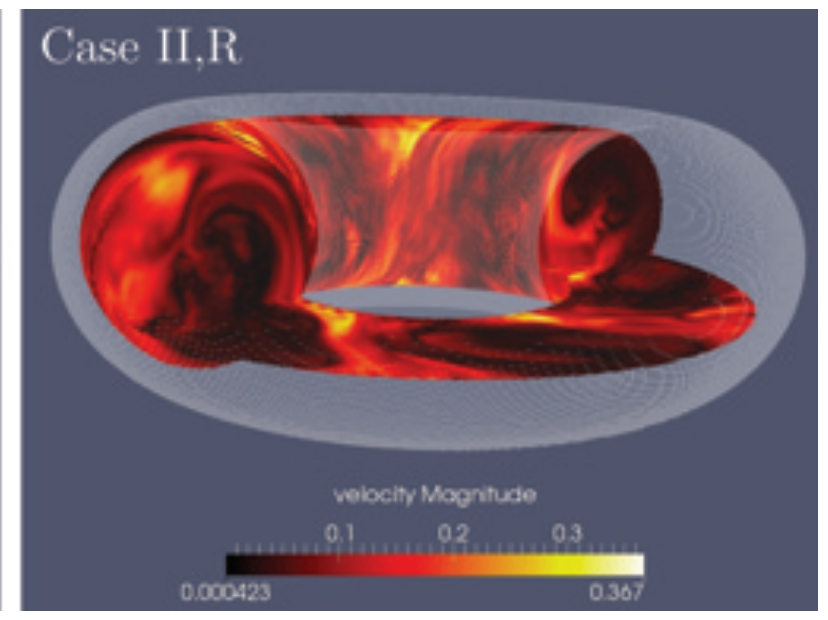

FIG. 5. Isosurfaces at $20 \%$ of the maximum toroidal velocity (red contours show positive velocity and blue contours negative) at steady state for Lundquist number $(M=2000)$. (Left) $B_{\text {tor }}=0.6$ and (Right) $B_{\text {tor }}=0.05$. 
Case I

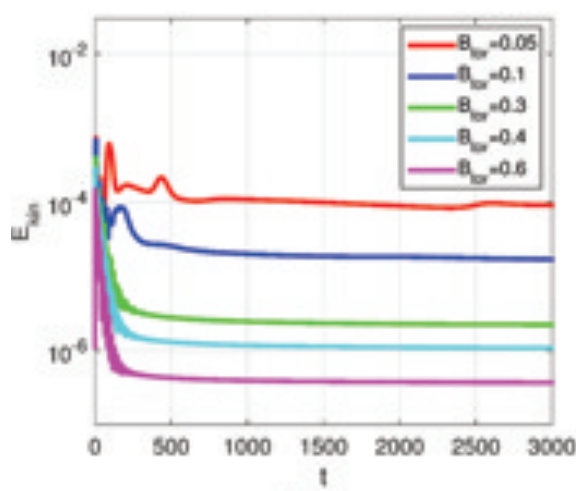

(a)

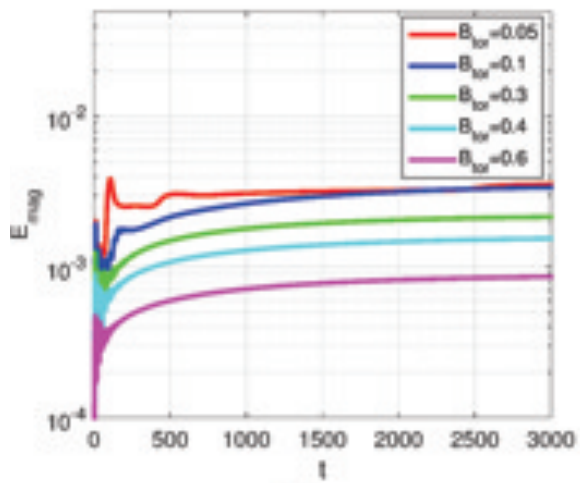

(c)

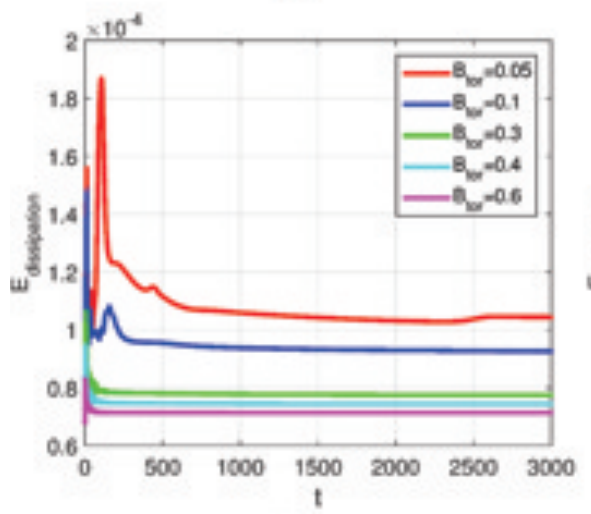

(e)

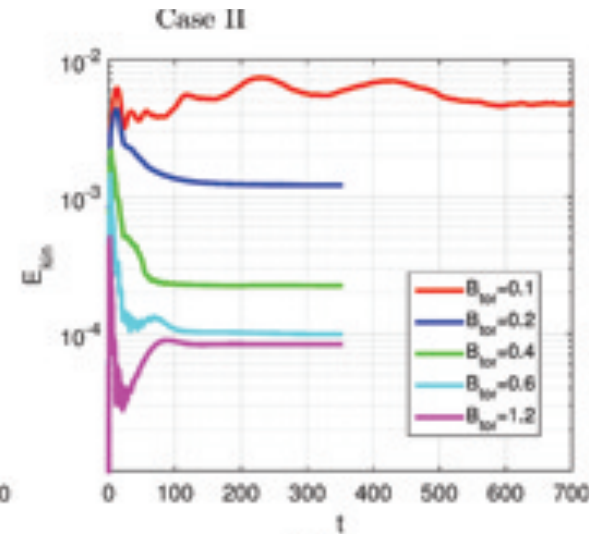

(b)

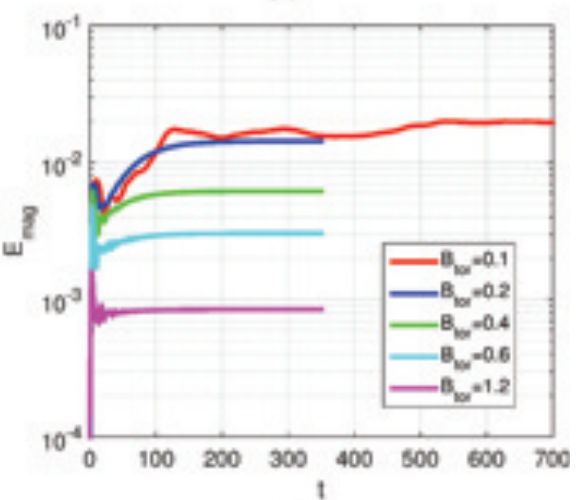

(d)

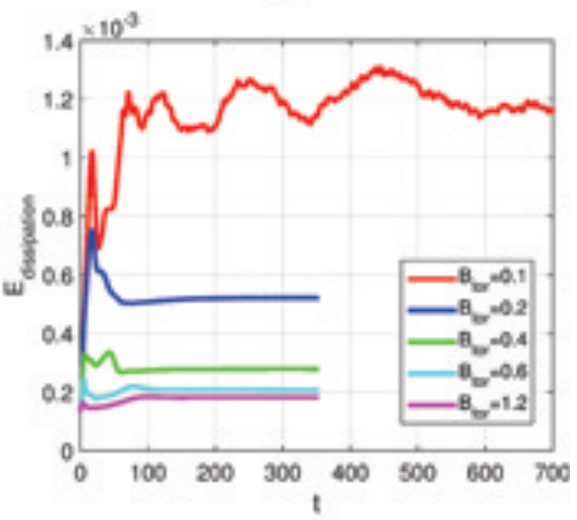

(f)
FIG. 6. The time evolution of the kinetic energy ((a) and (b)), the magnetic energy ((c) and (d)), and total dissipation of energy ((e) and (f)) at Lundquist number $M=2000$ for both uniform and non-uniform transport coefficients. The red curves in each graph, corresponding to $B_{t o r}=0.05$ and $B_{t o r}=0.1$ for case I and II, respectively, represent simulations in the RFP regime. The other curves correspond to the more quiescent tokamak regime.

\section{A. Characterization of the magnetic field and flow vizualisations}

Figure 2 shows the poloidal cross section of the profiles of the magnetic field. In the tokamak-regime (with $\left.B_{\text {tor }}>0.1\right)$, the velocity fluctuations are small, and at unity $\mathrm{Pr}$, magnetic fluctuations are so as well. The magnetic fields at steady state are thus close to the ones imposed by the external electric and magnetic fields only. In the RFP-regime (with $B_{\text {tor }} \leq 0.1$ ), the MHD instabilities lead to fluctuations of the magnetic field with an amplitude larger than $100 \%$ of the base field. In particular, the toroidal magnetic field is strongly increased in the plasma core while at the edge, it is strongly decreased. In the RFP community, this modification is often called the dynamo effect (e.g., Ref. 20), even though this nomenclature in the geophysical community is, in general, reserved for the generation of a magnetic field (of planets or stars) in the absence of strong initial magnetic fields. The resulting toroidal magnetic field thereby completely changes, to approach a poloidally symmetric shape, instead of the toroidally symmetric field in the tokamak case.

A more quantitative way to characterize these fields is by means of the safety-factor profiles. Figure 3 shows the profiles of the safety factor $q$ versus the normalized fluxfunction for two values of the toroidal magnetic field strength. If the toroidal magnetic field $B_{\text {tor }}$ is large enough, corresponding to the tokamak (T) regime, the modification of the initial magnetic field by the dynamic equilibrium velocity is small. For case II, T, the shape of the q-profile, is more parabolic than in the uniform-resistivity case, which is 


\section{Case I}

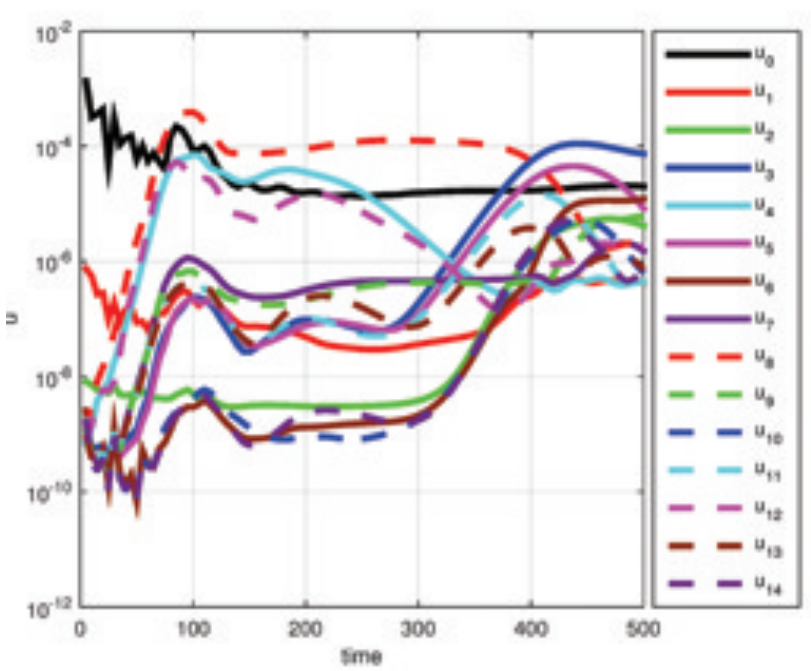

(a)

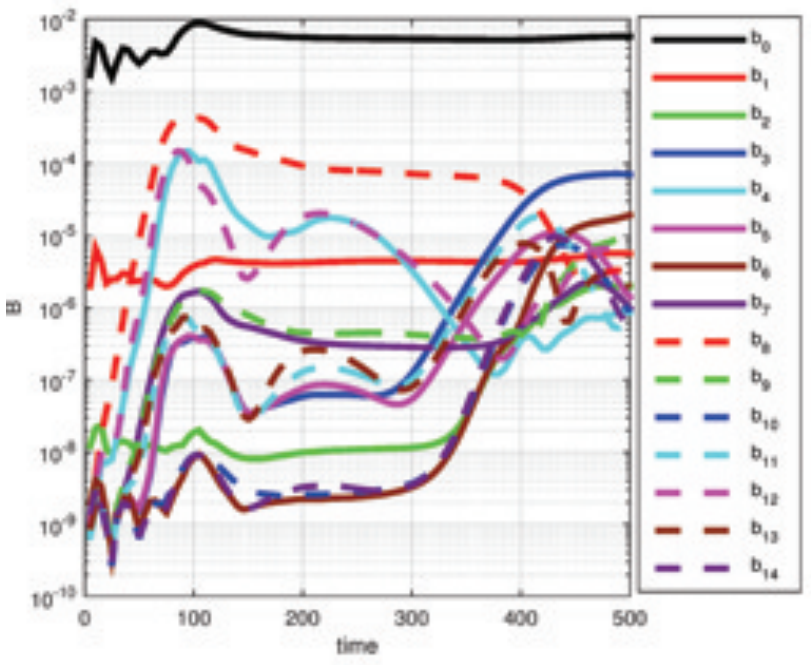

(c)
Case II

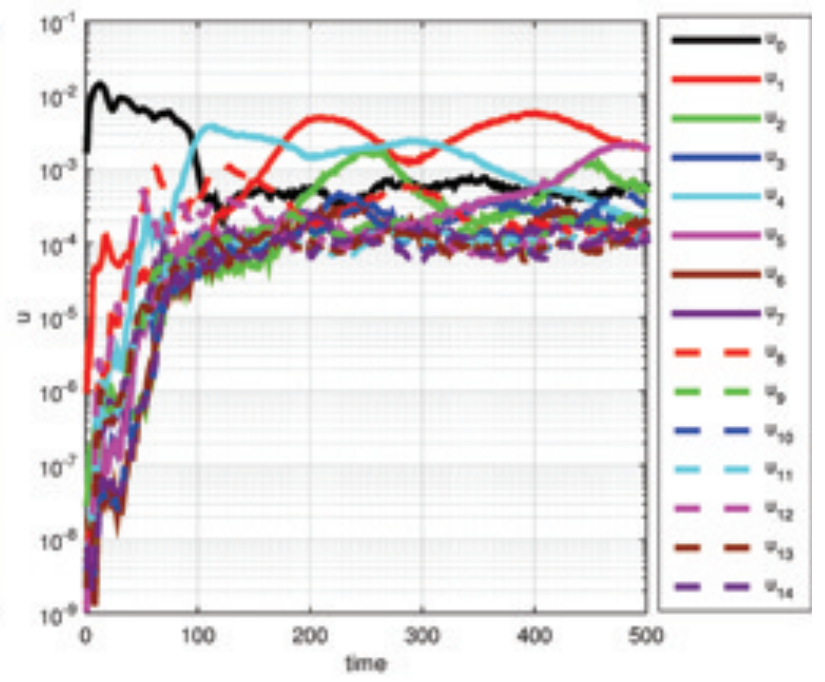

(b)

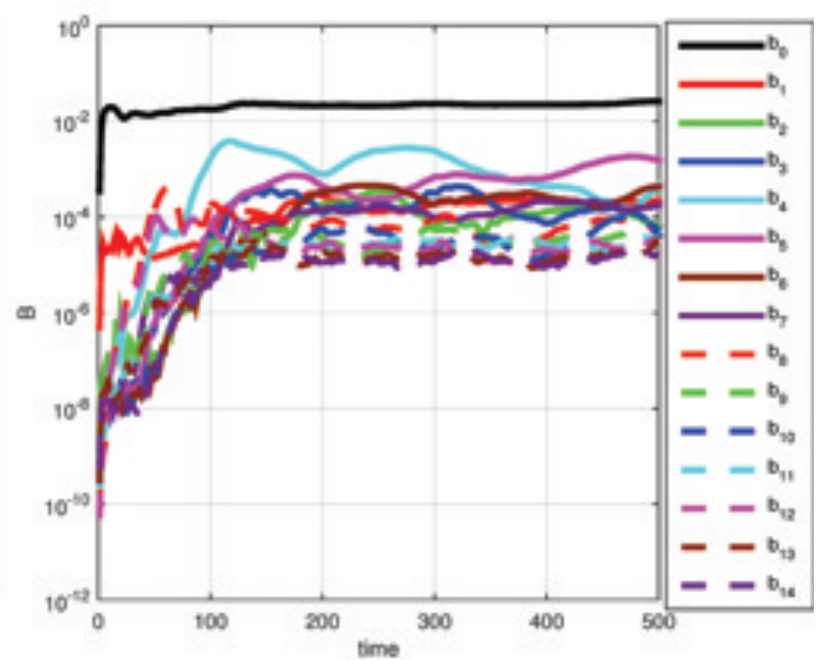

(d)

FIG. 7. The toroidal mode spectrum for the RFP regime for both case I and II over the time interval between $0<t<500$ of Fig. 6 for the velocity field ((a) and (b)) and the magnetic field ((c) and (d)).

a more realistic behaviour for fusion plasmas. ${ }^{21}$ Decreasing the toroidal magnetic field $B_{\text {tor }}$ under its threshold value triggers a chaotic plasma dynamics, characteristic of RFP devices. The resulting plasma movements change the q-profile importantly. The change of the q-profile in the RFP regime is strongest for case II, where the q-profile drops from a value of 0.5 in the core to a value near zero at the edge. For both cases I and II no complete reversal of the toroidal magnetic field is observed at the edge, although it approaches a zero toroidal magnetic field more closely at the edge. The reason of the difficulty of the achievement of the reversal of the toroidal magnetic field at the edge may be the incompressiblity assumed in the present approach. ${ }^{22}$ Taking into account compressibility of the plasma is, however, beyond the scope of the present investigation and would require the use of completely different numerical methods. However, even without a complete reversal, the dramatic change in the magnetic field configuration allows, already in the incompressible description, to investigate the self-organization of the toroidal plasma in the RFP regime.

In order to give an idea of the three-dimensional structure of the velocity-fields, Figure 4 shows the iso-surface of $20 \%$ of the maximum steady state toroidal velocity for case I,R and case I,T. If the toroidal magnetic field $B_{\text {tor }}$ is large enough, the dynamics of the magnetic field and the velocity field are not chaotic, and their fluctuations are small compared to those in the RFP-like regime. In the latter case, the plasma becomes unstable and the velocity field forms a helical structure winding around the toroidal axis. The details of this latter case with homogeneous transport coefficients are described in Ref. 6.

Vizualisations of the velocity magnitude for case I and II in the RFP regime are shown in Figure 5. In particular, the 

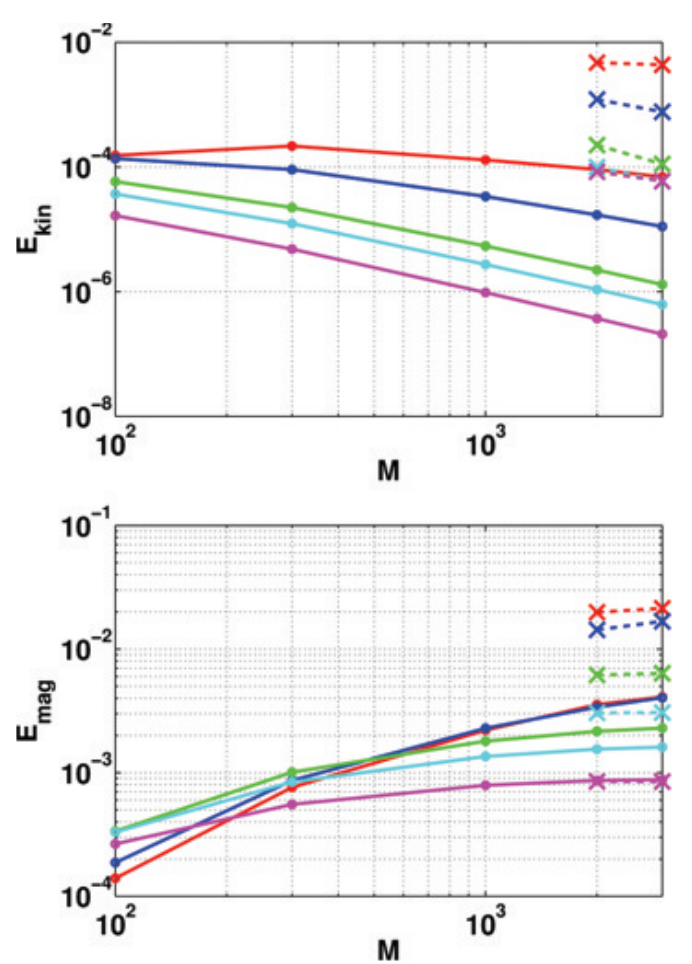

FIG. 8. Top: kinetic energy. Bottom: magnetic energy. Steady-state values versus Lundquist number. Colors are chosen as in Figure 6, the red curves corresponding to the RFP regime, the others corresponding to the tokamak regime. Solid lines correspond to case I (uniform viscosity and diffusivity), dashed lines to case II.

velocity gradients are more important in case II. This is related to the fact that the comparison of the two cases is done for a given equal current. In the case of a non-uniform resistivity profile, the current is more concentrated in the center of the domain, so that the local pinch ratio will be higher, which is in agreement with the lower value of the safety-factor in the center of the plasma (Figure 3). This generates a wider variety of unstable modes, ${ }^{1}$ as is reflected in the visualizations in Figure 5.

\section{B. Kinetic and magnetic energy and dissipation}

In the following, the magnetic energy corresponds to the perturbation part, i.e., we do not evaluate the contribution of the initially imposed magnetic field to the magnetic energy.
Figure 6 shows the time evolution of the kinetic energy and the magnetic energy for $M=2000$. In the tokamak regime $\left(B_{\text {tor }}>0.1\right)$, the energy evolution behaves similarly for all cases, representing only quantitative differences. However, in the RFP-regime $\left(B_{\text {tor }}<0.1\right)$, the energy evolution shows the signature of non-linear MHD activity with a non-monotonic evolution of the kinetic and magnetic energy up to $t=600$, after which a statistically steady state is reached. The noisy time-evolution of the dissipation suggests that in case II, the activity is turbulent. The time-evolution of the RFP state in case I seems to be less turbulent, with a smoother evolution of the energy and dissipation as a function of time.

A more refined view of the nonlinear dynamics underlying the RFP dynamics is obtained by monitoring the energy evolution of the individual modes constituting the flow. Figure 7 shows the time-evolution of the toroidal spectrum of the velocity field and the perturbation part of the magnetic field which are extracted from the simulation at $M=2000$ and $B_{\text {tor }}=0.05$ of Fig. 6 for a time-interval between $0<t$ $<500$ to that of case I. The evolution for case II is qualitatively quite similar in the sense that a wide spectrum of modes is excited. These modes are, however, fluctuating, reflecting the more turbulent behaviour of the simulation of case II. For both quantities (velocity and magnetic field), the spectrum shows the temporal evolution of a wide range of modes. A strong resemblance between the behaviour of the velocity and magnetic field modes is observed at long times, where an important number of modes with the same order of magnitude interacts. A dominant $n=0$ magnetic mode appears instantaneously, corresponding to the strongly modified magnetic field observed in Figure 2. The dominant velocity mode corresponds to $n=6$ for the present case, and an important $n=0$ subdominant velocity mode is observed, as was shown in Ref. 6. It is the $n=6$ velocity structure, flowing in both toroidal directions, which sustains - at long-times - the modified axisymmetric magnetic field typical of RFP dynamics.

Figure 8 shows the steady state values of the kinetic energy and the magnetic energy versus the Lundquist number. Increasing $M$ leads to a decrease in the kinetic energy roughly proportional to $M^{-1}$ for the tokamak regime. However, in the RFP-regime, the kinetic energy seems to decrease more gently with $M$. The fluctuating magnetic energy increases with $M$ until it saturates at a value depending on the pinch ratio. In the RFP regime, this increase is smaller, probably since more energy is contained in the
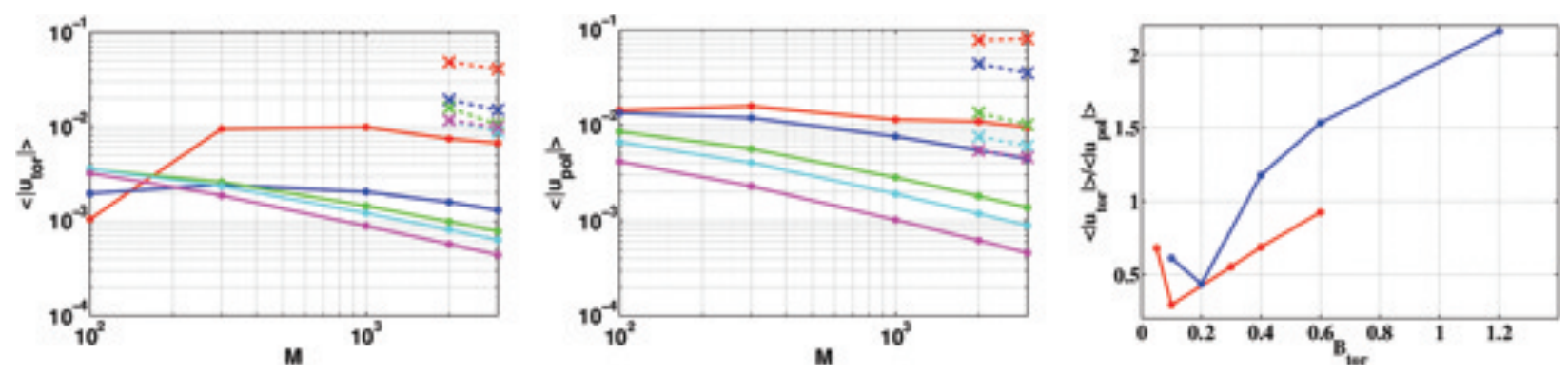

FIG. 9. Lundquist number dependence of the magnitudes of (a) the toroidal velocity, (b) the poloidal velocity. Colors in (a) and (b) are chosen as in Figure 6. (c) The ratio of the quantities in (a) and (b) as a function of the toroidal magnetic field for $M=2000$ for case I (blue) and case II (red). 


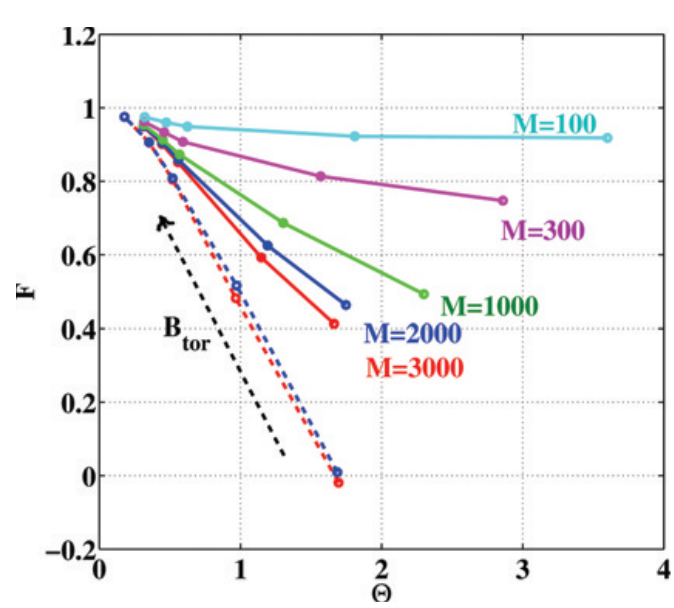

FIG. 10. Diagram of $F$ versus $\Theta$. Solid lines indicate the cases of uniform magnetic resistivity, and dashed lines are the cases of the magnetic resistivity profile $\eta(r)$.

velocity field. The values for case II, where the viscosity and diffusivity are a function of the minor radius, are more than an order of magnitude larger than the values for case I. The increased MHD activity in the core, due to the locally higher pinch ratio, can be held responsible for this.

\section{Toroidal and poloidal velocities and evolution of the reversal parameter.}

The kinetic energy of the MHD activity in Subsection III B can be decomposed into a component in the toroidal direction and a component in the poloidal plane. In all simulations, it was observed that the time and volume-averaged toroidal velocity is equal to zero, due to the up-down symmetry of the toroidal geometry. If this symmetry is broken, the resulting flow pattern can lead to non-zero angular momentum. ${ }^{16}$ This is not the case here. We will however be interested in the orientation of the flow pattern with respect to the toroidal axis, and we therefore compute the rms values of the toroidal velocity and of the velocity in the poloidal plane. Their Lundquist number dependence is shown in Figure 9. In the tokamak regime, both the toroidal and poloidal velocity are a decreasing function of the Lundquist number, while in the RFP-regime, the values seem to saturate. In the tokamak-regime, both velocity magnitudes are roughly proportional to $M^{-1}$. Figure 9(c) shows the evolution of the ratio of the toroidal velocity and the poloidal velocity, $\left\langle\left|u_{\text {tor }}\right|\right\rangle /\left\langle\left|u_{p o l}\right|\right\rangle$ as a function of $B_{\text {tor }}$ for both the tokamak and the RFP regime. It is observed that this ratio is an increasing function of $B_{t o r}$ for fixed $B_{p o l}$, in agreement with the observation that the velocity field tends to align with the magnetic field. ${ }^{16}$ Only in the RFP regime, this tendency is absent, due to the self-organization of the magnetic field. Again, the fact that for a given total current the current-channel is reduced by the resistivity profile leads to a modified flow geometry, as quantified by the ratio $\left\langle\left|u_{\text {tor }}\right|\right\rangle /\left\langle\left|u_{\text {pol }}\right|\right\rangle$.

A parameter that quantifies the modification of the magnetic field due to MHD activity is the reversal parameter

$$
F=\frac{\overline{B_{t o r}(a)}}{\left\langle B_{t o r}\right\rangle},
$$

with $a$ the minor radius. Figure 10 shows the diagram of the reversal parameter $F$ versus pinch ratio $\Theta$. In general, the non-uniform magnetic resistivity cases show lower values of the reversal parameter for a given pinch ratio $\Theta$. In the low Lundquist number limit (e.g., $M=100$ ), the decrease of $B_{\text {tor }}$ does not substantially modify $F$ although it increases the pinch ratio $\Theta$. This is different in the high Lundquist regimes ( $M>2000$ ), where by decreasing $B_{\text {tor }}$, the reversal parameter drops strongly. This shows that, if we identify the RFP regime by a drop in $F$ of, say, below 0.5 , one needs necessarily a high enough Lundquist number to trigger the RFP transition. This is in agreement with the observations in Refs. 1 and 2, who studied the onset of MHD instability in a periodic cylinder as a function of the Hartmann number and pinch ratio.

\section{CONCLUSIONS}

The present manuscript presented a comprehensive numerical study of the dynamics of incompressible, visco-resistive MHD in a toroidal geometry. The parameter-space was explored by varying the safety-factor (or pinch-ratio), and the Lundquist number. Both uniform and spatially variable viscosity and resistivity were considered. The resulting dynamics included dynamic equilibria for the quiescent, tokamak-like, regime, and the chaotic RFP-like regime. It was shown that for a given value of the Lundquist number, the kinetic and magnetic energy can vary over orders of magnitude when the pinch ratio is varied. The velocity magnitudes, decreasing approximately proportional to $M^{-1}$ for the tokamak cases, seem to decay more gently in the RFP regime, but more datapoints are needed to determine its exact dependence on the Lundquist number.

For a given total current, the velocities and magnetic fluctuations are more important by over an order of magnitude for the case where the transport-coefficients are varying as a function of the radius. Furthermore, the reversal parameter drops more importantly as a function of the pinch-ratio for this latter case. This last effect can be explained, at least in part by the non-uniformity of the local pinch-ratio, attaining larger values in the current channel for the case of non-uniform resisitivity.

Apart from these quantitative differences, no important qualitative differences seemed to be introduced by the nonuniform transport coefficients, justifying, in part, studies considering uniform transport coefficients, as long as no quantitative agreement with experiments is aimed for.

\section{ACKNOWLEDGMENTS}

We acknowledge the financial support from the French Research Agency (ANR), Project SiCoMHD, Contract ANR-11-BLAN-045. We are grateful to PCMS2I and computer center IDRIS (Project No. 22206), for computer resources. The interaction with David Montgomery and Kai Schneider is gratefully acknowledged.

${ }^{1} \mathrm{X}$. Shan and D. Montgomery, "On the role of the Hartmann number in magnetohydrodynamic activity," Plasma Phys. Controlled Fusion 35, 619 (1993).

${ }^{2}$ X. Shan and D. Montgomery, "Global searches of Hartmann-number-dependent stability boundaries," Plasma Phys. Controlled Fusion 35, 1019 (1993). 
${ }^{3}$ S. Cappello and D. F. Escande, "Bifurcation in viscoresistive MHD: The Hartmann number and the reversed field pinch," Phys. Rev. Lett. 85, 3838 (2000).

${ }^{4}$ M. Onofri, F. Malara, and P. Veltri, "Compressibility effects in the dynamics of the reversed-field pinch," Phys. Rev. Lett. 101(25), 255002 (2008).

${ }^{5}$ M. Onofri, F. Malara, and P. Veltri, "Effects of compressibility and heating in magnetohydrodynamics simulations of a reversed field pinch," Phys. Plasmas 16, 052508 (2009).

${ }^{6} \mathrm{~J}$. Morales, W. Bos, K. Schneider, and D. Montgomery, "The effect of toroidicity on reversed field pinch dynamics," Plasma Phys. Controlled Fusion 56, 095024 (2014).

${ }^{7}$ S. Cappello, D. Bonfiglio, D. Escande, S. Guo, I. Predebon, F. Sattin, M. Veranda, P. Zanca, C. Angioni, L. Chacón, J. Dong, X. Garbet, and S. Liu, "Equilibrium and transport for quasi-helical reversed field pinches," Nucl. Fusion 51, 103012 (2011)

${ }^{8}$ D. Bonfiglio, M. Veranda, S. Cappello, D. Escande, and L. Chacón, "Experimental-like helical self-organization in reversed-field pinch modeling," Phys. Rev. Lett. 111(8), 085002 (2013).

${ }^{9}$ A. Aydemir, "Shear flows at the tokamak edge and their interaction with edge-localized modes," Phys. Plasmas 14, 056118 (2007).

${ }^{10} \mathrm{~A}$. Aydemir, "Shear flows at the tokamak edge and their role in core rotation and the $L-H$ transition," Phys. Rev. Lett. 98, 225002 (2007).

${ }^{11}$ S. Pamela, G. Huysmans, and S. Benkadda, "Influence of poloidal equilibrium rotation in MHD simulations of edge-localized modes," Plasma Physics Controlled Fusion 52(7), 075006 (2010).

${ }^{12}$ J. Freidberg, "Ideal magnetohydrodynamic theory of magnetic fusion systems," Rev. Mod. Phys. 54, 801 (1982).
${ }^{13}$ D. Montgomery, H. Chen, and X. Shan, "Effects of uniform rotation on helically-deformed, resistive, magnetohydrodynamic equilibria," Plasma Phys. Controlled Fusion 33, 1871 (1991).

${ }^{14}$ J. Bates and D. Montgomery, "Toroidal visco-resisitive magnetohydrodynamic states contain vortices," Phys. Plasmas 5, 2649 (1998).

${ }^{15}$ L. Kamp and D. Montgomery, "Toroidal flows in resisitive magnetohydrodynamic states,” Phys. Plasmas 10, 157 (2003).

${ }^{16}$ J. Morales, W. Bos, K. Schneider, and D. Montgomery, "Intrinsic rotation of toroidally confined magnetohydrodynamics,” Phys. Rev. Lett. 109, 175002 (2012).

${ }^{17}$ J. Morales, W. Bos, K. Schneider, and D. Montgomery, "Magnetohydrodynamically generated velocities in confined plasma," Phys. Plasmas 22, 042515 (2015).

${ }^{18} \mathrm{P}$. Angot, C. Bruneau, and P. Fabrie, "A penalization method to take into account obstacles in viscous flows," Numer. Math. 81, 497 (1999).

${ }^{19}$ J. Morales, M. Leroy, W. Bos, and K. Schneider, "Simulation of confined magnetohydrodynamic flows with Dirichlet boundary conditions using a pseudo-spectral method with volume penalization," J. Comput. Phys. 274, 64 (2014).

${ }^{20}$ D. F. Escande, S. Cappello, F. D'Angelo, P. Martin, S. Ortolani, and R. Paccagnella, "Single helicity: A new paradigm for the reversed field pinch," Plasma Phys. Controlled Fusion 42, B243 (2000).

${ }^{21}$ X. Litaudon, Y. Sakamoto, P. De Vries, A. Salmi, T. Tala, C. Angioni, S. Benkadda, M. Beurskens, C. Bourdelle, M. Brix et al., "Core transport properties in JT-60U and JET identity plasmas," Nucl. Fusion 51, 073020 (2011).

${ }^{22}$ J. M. Finn, R. Nebel, and C. Bathke, "Single and multiple helicity Ohmic states in reversed-field pinches,” Phys. Fluids B 4, 1262-1279 (1992). 